DOI: $10.2478 /$ v10324-012-0019-y<smiles>C1C2CC1C2</smiles>

VERSITA
Analele Universităţii de Vest,

Timişoara

Seria Matematică - Informatică

L, 2, (2012), 117- 123

\title{
Partly Interpolation Space in the Multidimensional Case
}

Ilie Stan

\begin{abstract}
We give a necessary and sufficient condition for an intermediate Banach space $A$ with respect to the Banach $N+1-$ tuple $\vec{A}$ to be partly interpolation space. We also establish some properties on partly interpolation spaces.
\end{abstract}

AMS Subject Classification (2000). 46M35.

Keywords. Interpolation spaces, partly spaces.

\section{Introduction}

It is well known that exist an intermediate space with respect to the triple $\left(L_{1}, L_{p}, L_{\infty}\right), 1<p<\infty$, which is partly interpolation space but not an interpolation space (see [3], [4],[5], [6]).

Working with Banach couples, Dimitriev [4] and Pustylnik [6] have been introduce and studied the functions $\psi, \rho$ which describe the "position" of the intermediate space within the Banach couple. They show that a necessary and sufficient condition for an intermediate Banach space $A$ with respect to the Banach couple $\vec{A}$ to be a party interpolation space is that for some $C>0$ it holds

$$
\psi(t) \leq C \rho(t), \quad \text { for all } t>0 .
$$

This research was continued by Cobos and Romero [3] in the context of $N_{-}$ tuples of Banach spaces, by using the $K$-and $J$-functionals associated to a 
convex polygon in the plane. They show that the corresponding inequality (1.1) is a characterization of partly interpolation spaces only if $N=3$.

Our aim is to study this problem in the context of $(N+1)$-tuples $(N \geq 2)$ of Banach spaces, by using Sparr's [7] $K$-and $J$-functionals. In this case the corresponding inequality (1.1) is a characterization of partly interpolation space for every finite $N \geq 2$.

\section{Preliminaries}

Let $\vec{A}=\left(A_{0}, \ldots, A_{N}\right)$ be a Banach $N+1$-tuple, that is, a family of $N+1$ Banach spaces $A_{i}$ all of them continuously embedded in a common Hausdorff topological vector space $\mathcal{U}$ (i.e. $A_{i} \hookrightarrow \mathcal{U}$ ). For each $\bar{t}=\left(t_{1}, \ldots, t_{N}\right)>0$, Sparr's $K$-and $J$-functionals are defined by

$$
K(\bar{t}, a, \vec{A})=\inf \left\{\left\|a_{0}\right\|_{A_{0}}+\sum_{i=1}^{N} t_{i}\left\|a_{i}\right\|_{A_{i}}: a=\sum_{i=0}^{N} a_{i}, a_{i} \in A_{i}\right\},
$$

for each $a \in \Sigma(\vec{A})=A_{0}+\cdots+A_{N}$

and

$$
J(\bar{t}, a, \vec{A})=\max _{1 \leq i \leq N}\left(\|a\|_{A_{0}}, t_{i}\|a\|_{A_{i}}\right)
$$

for each $a \in \Delta(\vec{A})=A_{0} \cap \cdots \cap A_{N}$.

A linear operator $T$ in $\Sigma(\vec{A})$ is called a bounded linear operator on a Banach $N+1$-tuple $\vec{A}$ if $T$ maps $A_{i}$ into $A_{i}(i=0, \ldots, N)$ boundedly. Let $\mathcal{L}(\vec{A}, \vec{A})$ stand for the set of all such operators. $\mathcal{L}(\vec{A}, \vec{A})$ is a Banach space with respect to the norm

$$
\|T\|_{\vec{A}, \vec{A}}=\max _{0 \leq i \leq N}\left(\|T\|_{A_{i}, A_{i}}\right) .
$$

A Banach space $A$ is said to be an intermediate space with respect to $\vec{A}$ if

$$
\Delta(\vec{A}) \hookrightarrow A \hookrightarrow \Sigma(\vec{A}) .
$$

If $B$ is an intermediate space with respect to $\vec{A}$ with $B \subset A$, then $B \hookrightarrow A$. An intermediate space $A$ is an interpolation space if, given any $T \in \mathcal{L}(\vec{A}, \vec{A})$, the restriction of $T$ to $A$ defines a bounded operator from $A$ into itself. Moreover, by the closed graph theorem, there exists a constant $C=C(A, \vec{A})$ such that

$$
\|T\|_{A, A} \leq C\|T\|_{\vec{A}, \vec{A}}, \quad \text { for all } T \in \mathcal{L}(\vec{A}, \vec{A}) .
$$


We say that the intermediate space $A$ is a partly interpolation space, or a rank-one interpolation space, if (2.1) holds for all $T \in \mathcal{L}(\vec{A}, \vec{A})$ of rank-one (i.e. of the special form: $T=f \otimes a$, where $f \in(\Sigma(\vec{A}))^{*}$ and $\left.a \in \Delta(\vec{A})\right)$. We shall denote the set of partly interpolation spaces for the $N+1$-tuple $\vec{A}$ by $R 1-\operatorname{Int}(\vec{A})$.

Let $A$ be an intermediate space with respect to $\vec{A}=\left(A_{0}, \ldots, A_{N}\right)$. For each $\bar{t}=\left(t_{1}, \ldots, t_{N}\right)>0$ set

$$
\psi(\bar{t})=\psi(\bar{t} ; A, \vec{A}):=\sup \left\{K(\bar{t}, a, \vec{A}):\|a\|_{A}=1\right\}
$$

and

$$
\rho(\bar{t})=\rho(\bar{t} ; A, \vec{A}):=\inf \left\{J(\bar{t}, a, \vec{A}): a \in \Delta(\vec{A}),\|a\|_{A}=1\right\} .
$$

To each intermediate space $A$ we associate the Gagliardo completion of $A$ (denoted by $\left.\widetilde{A}^{\Sigma(\vec{A})}\right)$, consists of all those $a \in \Sigma(\vec{A})$ for which there exists a bounded sequence $\left(a_{n}\right)_{n \in \mathbb{N}}$ in $A$ which converges to $a$ in $\Sigma(\vec{A})$. It is normed by

$$
\|a\|_{\widetilde{A}^{\Sigma(\vec{A})}}=\inf _{\left(a_{n}\right)}\left\{\sup _{n \in \mathbb{N}}\left(\left\|a_{n}\right\|_{A}\right)\right\} .
$$

It is easy to show (see [1]) that

$$
\|a\|_{\widetilde{A}_{0} \Sigma(\vec{A})}=\lim _{\bar{t} \rightarrow \bar{\infty}} K(\bar{t}, a, \vec{A}) .
$$

\section{$3 \quad$ Partly interpolation spaces}

Theorem 3.1. Let $\vec{A}=\left(A_{0}, \ldots, A_{N}\right)$ be a Banach $N+1$-tuple and let $A$ be an intermediate spaces with respect to $\vec{A}$. Then $A$ is party interpolation space if and only if there exists a pozitive constant $C=C(A, \vec{A})$ such that

$$
\psi(\bar{t}) \leq C \rho(\bar{t}), \text { for all } \bar{t}=\left(t_{1}, \ldots, t_{N}\right)>0 .
$$

Proof. Assume that $A$ is partly interpolation space and let $C$ be the constant from (2.1) for one-dimensional operators. We prove that, for any $a \in A$ and any $b \in \Delta(\vec{A})$, we have

$$
\frac{K(\bar{t}, a, \vec{A})}{\|a\|_{A}} \leq C \frac{J(\bar{t}, b, \vec{A})}{\|b\|_{A}}, \text { for all } \bar{t}>0 .
$$

This can be done using ideas of [3]. 
For $a$ given $a \in A$ we take the functional $f \in(\Sigma(\vec{A}))^{*}$ such that $f(a)=$ $K(\bar{t}, a, \vec{A})$ and $|f(x)| \leq K(\bar{t}, x, \vec{A})$ for any $x \in \Sigma(\vec{A})$. Then, we have

$$
\|f\|_{A_{0}^{*}}=\sup _{x \in A_{0}} \frac{|f(x)|}{\|x\|_{A_{0}}} \leq \sup _{x \in A_{0}} \frac{K(\bar{t}, x, \vec{A})}{\|x\|_{A_{0}}} \leq \sup _{x \in A_{0}} \frac{\|x\|_{A_{0}}}{\|x\|_{A_{0}}}=1
$$

and, for $i=\overline{1, N}$

$$
\|f\|_{A_{i}^{*}}=\sup _{x \in A_{i}} \frac{|f(x)|}{\|x\|_{A_{i}}} \leq \sup _{x \in A_{i}} \frac{K(\bar{t}, x, \vec{A})}{\|x\|_{A_{i}}} \leq \sup _{x \in A_{i}} \frac{t_{i}\|x\|_{A_{i}}}{\|x\|_{A_{i}}}=t_{i} .
$$

Take any $b \in \Delta(\vec{A})$ and consider the one-dimensional operator $T x=f(x) b$. Then, for $i \in \overline{0, N}$, we have

$$
\|T\|_{A_{i}, A_{i}}=\sup _{x \in A_{i}} \frac{\|T x\|_{A_{i}}}{\|x\|_{A_{i}}}=\|f\|_{A_{i}^{*}}\|b\|_{A_{i}} .
$$

Since $A$ is partly interpolator space, from (2.1) we get that

$$
\begin{gathered}
\|T a\|_{A} \leq C \max _{0 \leq i \leq N}\left(\|T\|_{A_{i}, A_{i}}\right)\|a\|_{A}=C \max _{0 \leq i \leq N}\left(\|f\|_{A_{i}^{*}}\|b\|_{A_{i}}\right)\|a\|_{A} \leq \\
\leq C \max \left(\|b\|_{A_{0}}, t_{1}\|b\|_{A_{1}}, \ldots, t_{N}\|b\|_{A_{N}}\right)\|a\|_{A}=C J(\bar{t}, a, \vec{A})\|a\|_{A} .
\end{gathered}
$$

But, from the definition of $T$ we get

$$
\|T a\|_{A}=|f(a)|\|b\|_{A}=K(\bar{t}, a, \vec{A})\|b\|_{A} .
$$

So, we obtain (3.2). From (3.2) follows (3.1).

Conversely, suppose that (3.1) holds. Take any one-dimensional operator $T x=f(x) b$ (i.e. take any $f \in(\Sigma(\vec{A}))^{*}$ and any $\left.b \in \Delta(\vec{A})\right)$. For any $a \in A$, using (3.1) we get

$$
\begin{gathered}
\|T a\|_{A}=|f(a)|\|b\|_{A} \leq|f(a)| \frac{J(\bar{t}, b, \vec{A})}{\rho(\bar{t})} \leq C|f(a)| \frac{J(\bar{t}, b, \vec{A})}{\psi(\bar{t})} \leq \\
\leq C|f(a)| \frac{J(\bar{t}, b, \vec{A})}{K(\bar{t}, a, \vec{A})}\|a\|_{A} .
\end{gathered}
$$

Let $a=\sum_{i=0}^{N} a_{i}$, with $a_{i} \in A_{i}$ a decomposition of a such that:

$$
\left\|a_{0}\right\|_{A_{0}}+\sum_{i=1}^{N} t_{i}\left\|a_{i}\right\|_{A_{i}} \leq 2 K(\bar{t}, a, \vec{A}) .
$$


Then

$$
\begin{gathered}
\|T a\|_{A} \leq 2 C \frac{\sum_{i=0}^{N}\left|f\left(a_{i}\right)\right|}{\left\|a_{0}\right\|_{A_{0}}+\sum_{i=1}^{N} t_{i}\left\|a_{i}\right\|_{A_{i}}} J(\bar{t}, b, \vec{A})\|a\|_{A} \leq \\
\leq 2 C(N+1) \max \left(\frac{\left|f\left(a_{0}\right)\right|}{\left\|a_{0}\right\|}, \frac{\left|f\left(a_{1}\right)\right|}{t_{1}\left\|a_{1}\right\|_{A_{1}}}, \ldots, \frac{\left|f\left(a_{N}\right)\right|}{t_{N}\left\|a_{N}\right\|_{A_{n}}}\right) J(\bar{t}, b, \vec{A})\|a\|_{A} \leq \\
\leq 2 C(N+1) \max \left(\|f\|_{A_{0}^{*}}, \frac{\|f\|_{A_{1}^{*}}}{t_{1}}, \ldots, \frac{\|f\|_{A_{N}^{*}}}{t_{N}}\right) \cdot \\
\cdot \max \left(\|b\|_{A_{0}}, t_{1}\|b\|_{A_{1}}, \ldots, t_{N}\|b\|_{A_{N}}\right)\|a\|_{A} .
\end{gathered}
$$

Now, we put $t_{i}=\frac{\|f\|_{A_{i}^{*}}}{\|f\|_{A_{0}^{*}}}, i=1,2, \ldots, N$. Then

$$
\|T a\|_{A} \leq 2 C(N+1) \max _{0 \leq i \leq N}\left(\|f\|_{A_{i}^{*}}\|b\|_{A_{i}}\right)\|a\|_{A}=2(N+1) C\|T\|_{\vec{A}, \vec{A}}\|a\|_{A} .
$$

Proposition 3.2. Let $\vec{A}=\left(A_{0}, \ldots, A_{N}\right)$ be a Banach $(N+1)$-tuple and let $A$ be a partly interpolation space with respect to $\vec{A}$.

a) If $\lim _{\bar{t} \rightarrow \bar{\infty}} \frac{1}{\rho(\bar{t})}>0$ then $A \hookrightarrow \widetilde{A}_{0}^{\Sigma((\vec{A})}$.

b) If $\lim _{\bar{t} \rightarrow \overline{0}} \psi(\bar{t})>0$ then $\overline{\Delta(\vec{A})^{A}} \hookrightarrow A$.

Proof. a) Suppose that $\lim _{\vec{t} \rightarrow \bar{\infty}} \frac{1}{\rho(\bar{t})}>0$. Then, by (3.1) we have $\lim _{\bar{t} \rightarrow \bar{\infty}} \psi(\bar{t})=$ $C_{1}<\infty$. It follows that for each $a \in A$ we have

$$
\|a\|_{\widetilde{A}_{0} \Sigma(\vec{A})}=\lim _{\vec{t} \rightarrow \bar{\infty}} K(\bar{t}, a, \vec{A}) \leq \lim _{\bar{t} \rightarrow \bar{\infty}} \psi(\bar{t})\|a\|_{A}=C_{1}\|a\|_{A}
$$

and so $A \hookrightarrow \widetilde{A}_{0}^{\Sigma(\vec{A})}$.

b) Suppose that $\lim _{\bar{t} \rightarrow \overline{0}} \psi(\bar{t})>0$. Then, by $(3.1)$ we have $\lim _{\bar{t} \rightarrow \overline{0}} \rho(\bar{t})=C_{2}>0$. It follows that $C_{2} \leq \frac{J(\bar{t}, a, \vec{A})}{\|a\|_{A}}$, for all $\bar{t}=\left(t_{1}, \ldots, t_{N}\right)>0$, and all $a \in \Delta(\vec{A})$. Therefore

$$
\|a\|_{A} \leq \frac{1}{C_{2}} \max \left(\|a\|_{A_{0}}, t_{1}\|a\|_{A_{1}}, \ldots, t_{N}\|a\|_{A_{N}}\right) .
$$


Now, we put $t_{i}=\frac{\|a\|_{A_{0}}}{\|a\|_{A_{i}}},(i=1,2, \ldots, N)$. This implies

$$
\|a\|_{A} \leq \frac{1}{C_{2}\|a\|_{A_{0}}}, \text { for all } a \in \Delta(\vec{A})
$$

and, consequently $\overline{\Delta(\vec{A})} A_{0} \hookrightarrow A$.

Proposition 3.3. Let $\vec{A}=\left(A_{0}, \ldots, A_{N}\right)$ and $\vec{B}=\left(B_{0}, \ldots, B_{N}\right)$ be two Banach $N+1$-tuples. Then

$$
R 1-\operatorname{Int}(\vec{A}) \subset R 1-\operatorname{Int}(\vec{B}) \Leftrightarrow A_{i} \in R 1-\operatorname{Int}(\vec{B}),(i=0, \ldots, N) .
$$

Proof. The implication $\Rightarrow$ is trivial. Conversely, suppose that $A_{i} \in R 1-$ $\operatorname{Int}(\vec{B}),(i=0, \ldots, N)$. Let $A \in R 1-\operatorname{Int}(\vec{A})$ and the one-dimensional operator $T \in \mathcal{L}(\vec{B}, \vec{B})$. Then, by $(2.1)$ we have

$$
\|T\|_{A_{i}, A_{i}} \leq C\|T\|_{\vec{B}, \vec{B}} \quad(i=-, 1, \ldots, N) .
$$

It follows that

$$
\|T\|_{A, A} \leq C\|T\|_{\vec{A}, \vec{A}} \leq C\|T\|_{\vec{B}, \vec{B}} .
$$

Then $A \in R 1-\operatorname{Int}(\vec{B})$.

Proposition 3.4. Let $\vec{A}=\left(A_{0}, \ldots, A_{N}\right)$ be a Banach $N+1$-tuple and let $A$ be a party interpolation space with respect to $\vec{A}$.

a) If $A \not \subset \underline{\bar{A}_{i} \text { then }} A_{0} \cap \ldots A_{i-1} \cap A_{i+1} \cap \ldots A_{N} \subset A,(i=0, \ldots, N)$.

b) If $A \not \subset \overline{\sum_{\substack{k=0 \\ k \neq i}}^{N} A_{k}}$ then $A_{i} \subset A,(i=0, \ldots, N)$.

Proof. a) If $A \not \subset \bar{A}_{i}$, there exists $a$ such that:

$$
0 \neq a \in A, \quad a \notin \bar{A}_{i} \quad(i=0, \ldots, N) .
$$

Then, according to the Hahn-Banach theorem, there exists a linear functional $f \in(\Sigma(\vec{A}))^{*}$ for which

$$
\left.f\right|_{A_{i}}=0, \quad f(a)=1 .
$$

Let $y$ be an arbitrary fixed element of $A_{0} \cap \cdots \cap A_{i-1} \cap A_{i+1} \cap \cdots \cap A_{N}$. We put $T:=f \otimes y$. Then $T \in \mathcal{L}(\vec{A}, \vec{A})$ and $y=T(a) \in A$.

The proof $(b)$ is similar.

Corollary 3.5. If for every $i \in\{0,1, \ldots, N\} A \not \subset \overline{\sum_{\substack{k=0 \\ k \neq i}}^{N} A_{k}}$ then $A=\Sigma(\vec{A})$. 


\section{References:}

[1] C. Bennett and R. Sharpley, Interpolation of Operators, Academic Press, Boston, 1988.

[2] F. Cobos, M. Cwikel, and Matos. P., Best possible compactness results of LionsPeetre type, Proc. Edinb. Math. Soc., 44, (2001), 153-172.

[3] F. Cobos and R. Romero, Lions-Peetre type compactness results for several Banach spaces, Mathematical Inequalities $\mathscr{E}$ Applications, 7, number 4, (2004), 557-571.

[4] A. A. Dmitriev, The interpolation of one-dimensional operators, Anal. i Prilozen, 11,(in Russian), (1973), 31-43.

[5] S. G. Krein, J. I. Petunin, and E. M. Semenov, Interpolation of Linear Operators, Amer. Math. Soc. Providence, R. I., 1982.

[6] E. I. Pustylnik, Embedding functions and their role in interpolation theory, 1, (1996), $305-325$.

[7] G. Sparr, Interpolation of several Banach spaces, Ann. Mat. Pura Appl., 99, (1974), $247-316$.

Ilie Stan

Department of Mathematics, West University of Timişoara

Blvd. V. Pârvan 4

Timişoara 300223, Timiş, Romania

E-mail: stan@math.uvt.ro

Received: 15.03 .2012

Accepted: 3.09 .2012 